\title{
évaluation des déformations des piliers par émission acoustique dans une carrière souterraine
}

\author{
evaluation of pillars deformation by acoustic emission \\ in an underground quarry
}

\author{
M.C. Reymond \\ Chargée de Recherche, C.N.R.S."
}

\section{Résumé}

L'évolution des déformations et de l'émission acoustique au cours d'une année dans une carrière souterraine chargée en surface par des remblais est décrite. Les déformations des piliers s'accompagne d'émission acoustique qui peut être interprétée comme la formation et la propagation de fissures. Les résultats des mesures de déformations et d'émission acoustique sont en corrélation et indiquent deux mécanismes de rupture.

\section{Abstract}

This paper describe for one year measurements of the deformation behaviour and Acoustic Emission in an underground quarry loaded by a pressure. The pillar deformation is accompanied by the A.E. as a result of the formation and growth of the cracks. The results of Acoustic Emission and deformation measurements are in agreement and indicate two failure mechanisms. 


\section{INTRODUCTION}

La complexité des phénomènes qui interviennent en mécanique des roches est telle qu'il est très difficile de prévoir le comportement d'une carrière à partir de la seule connaissance de quelques caractéristiques de la roche.

La surveillance acoustique constitue dans ce domaine un outil très utile : elle est fondée sur le fait qu'un effondrement ne survient pratiquement jamais brutalement. Il est précédé par des ébranlements de différentes natures pouvant être détectés par des accéléromètres. Ces émissions acoustiques sont dues aux légers déplacements de la roche qui se produisent sous l'effet d'un changement d'équilibre des contraintes.

Des scientifiques ont réalisé des approches intéressantes de ces phénomènes à laide de la technique acoustique: HARDY (1975), ANTSYFEROV (1974), JAROSZEWSKA (1982), REYMOND (1984), LASOCKI (1982).

L'objectif de cette étude est, d'une part de mettre en évidence ces ébranlements par l'émission acoustique et, d'autre part de tenter de relier ceux-ci à l'évolution des déformations du massif.

\section{PRÉSENTATION DE LA CARRIÈRE}

Le Laboratoire des Ponts-et-Chaussées, associé à l'Institut de Physique du Globe et au C.N.R.S., a été amené à suivre le comportement d'une carrière souterraine abandonnée de calcaire grossier soumise à une surcharge de remblais en surface à partir du mois de novembre 1984 (1). Il s'agit d'une ancienne exploitation selon la méthode des chambres et piliers.

Une reconnaissance préliminaire a donc été entreprise avant le remblayage afin de mieux connaitre les caractéristiques géotechniques de la zone composée de chambres et de piliers de section variable $(2,50 \mathrm{~m} \times 2,50 \mathrm{~m}$ ou $7,50 \mathrm{~m} \times 5 \mathrm{~m})$

Dans un premier temps, une campagne géologique a permis de déceler la présence d'un réseau de fractures orientées ouest-est, de nombreuses chutes de toit et des zones de bourrages de secteur de toit particulièrement humides (figure 1).

Dans un second temps une campagne de sondage a été réalisée dans le but de connaître les caractéristiques mécaniques à partir d'échantillons prélevés dans le toit et dans les piliers. Ces paramètres analysés par la section de géologie des matériaux du L.C.P.C. à Paris, à partir d'éprouvettes saturées en eau et testées en compression attestent une hétérogénéité importante de la roche (Tableau I).

(1) Laboratoire des Ponts-et-Chaussées (Laboratoire Régional de Lyon), Laboratoire des Ponts-et-Chaussées (Laboratoire Régional de l'Ouest Parisien-Trappes). Un article détaillé sera publié prochainement sur cette expérience dans la R.F.G.

\begin{tabular}{|l|}
\hline $\begin{array}{l}\text { Résistance à la compression simple sur } \\
\text { des éprouvettes saturées d'eau } \\
\mathrm{Rc}=4,5 \text { à } 15 \mathrm{MPa}\end{array}$ \\
\hline Module $\mathrm{E}=4600$ à $12000 \mathrm{MPa}$ \\
\hline Porosité $\mathrm{n}=28,7$ à $42,2 \%$ \\
\hline $\begin{array}{l}\text { Célérité des ondes longitudinales } \\
\mathrm{C}_{\ell}=2000 \text { à } 3100 \mathrm{~m} / \mathrm{s}\end{array}$ \\
\hline \begin{tabular}{l} 
Degré de saturation en eau : \\
toit : $\mathrm{Sr}=85$ à $100 \%$ \\
piliers : $\mathrm{Sr}=60$ à $70 \%$ \\
blocs détachés du massif : $\mathrm{Sr}=50$ à $55 \%$ \\
\hline
\end{tabular} \\
\hline
\end{tabular}

Tableau I. - Résumé des informations à partir d'essais en compression sur des éprouvettes calcaires : résistance à la compression simple, module d'élasticité, porosité, célérité des ondes. Tableau 1. - Summary of informations deduced from limestone specimens in compression tests : failure load, young modulus, porosity, compression wave velocity.

Dans un troisième temps différentes mesures ont été mises en place afin de suivre les effets du chargement sur quelques piliers: mesures des contraintes, des déformations, de la vitesse du son, mesures électriques et sismo-électriques; seuls seront pris en considération dans cet article les résultats sismo-acoustiques en relation avec les extensomètres et les variations de contraintes.

Les valeurs des contraintes mesurées avant le chargement à l'aide d'un vérin plat sur 11 piliers révèlent une répartition non uniforme des contraintes : par exemple des valeurs très différentes ont été relevées sur le pilier 108 , face $113(2,5 \mathrm{MPa})$ et sur le pilier 108 , face 107 $(4,37 \mathrm{MPa})$ (Tableau II).

\section{DESCRIPTION SOMMAIRE DES TECHNIQUES UTILISÉES}

\subsection{Extensométrie}

Des extensomètres ont été implantés au quart et au milieu des piliers 36,108 et 37 afin de permettre de suivre les déformations de plus grande amplitude pendant la période du 18 janvier 1985 au 29 décembre 1985. Le relevé des mesures était effectué trois fois par semaine par M. THORIN du Laboratoire Régional de l'Ouest Parisien.

\subsection{Chaîne acoustique}

La chaîne acoustique enregistrait les événements de façon continue; elle se composait (figure 2a) :

- d'un accéléromètre de sensibilité $60 \mathrm{mV} / \mathrm{g}$ (réponse plate $2 \mathrm{~Hz}$ à $16 \mathrm{kHz}$ ), vissé sur une tige en 


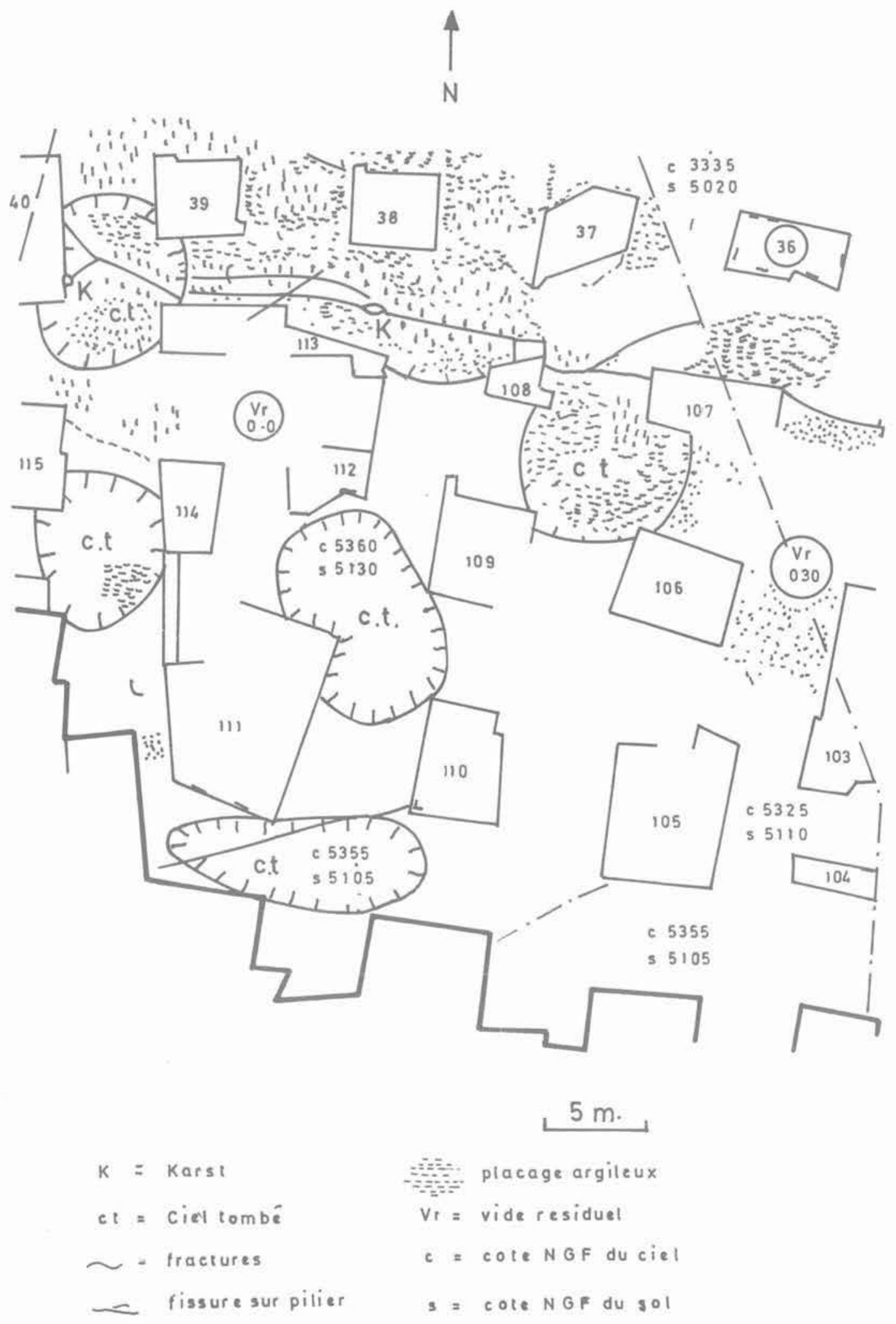

Fig. 1. - Schéma de la carrière souterraine de calcaire ou vue en plan du projet concernant le site expérimental devant être fissuré, montrant la position des piliers et la localisation du capteur (pilier 36). Fig. 1. - Underground limestone quarry contours or a plan view of the experimental rockbreaking project site showing the position of the pillars and the location of detector (pillar 36). 


\begin{tabular}{|c|c|c|c|c|c|c|}
\hline Piliers & $\begin{array}{l}\text { Co } \\
\text { Etat } \\
\text { initial } \\
\mathrm{MPa}\end{array}$ & $\begin{array}{l}\text { intes } \\
\text { Ėtat } \\
\text { au } 18 / 10 \\
\mathrm{MPa}\end{array}$ & $\begin{array}{c}\text { Coefficient de } \\
\text { corrélation de } \\
\text { Bravais-Pearson } \\
\text { r }\end{array}$ & \multicolumn{2}{|c|}{$\begin{array}{c}\text { Droites de régression } \\
(y=\text { déformations }) \\
(\alpha=\text { contraintes })\end{array}$} & $\begin{array}{l}\text { Observations } \\
\text { corrélation } \\
\text { en fonction }\end{array}$ \\
\hline P 36 , face 52 & 1,45 & 1,7 & & & & \\
\hline P 36 , face 107 & & & -.89 & $y=-0,000$ & $\sigma+25,54$ & du temps \\
\hline P 37 , face 51 & 2,65 & 4,75 & -.61 & $y=-0,006$ & $\sigma+19,4$ & de la charge \\
\hline P 37, face 108 & & & -.70 & $y=-0,000$ & $\sigma+23,04$ & du temps \\
\hline P 108 , face 113 & 2,56 & 4,15 & -.77 & $y=-0,005$ & $a+25,05$ & de la charge \\
\hline P 108, face 107 & 4,37 & & -.86 & $y=-0,001$ & $\sigma+24,27$ & du temps \\
\hline P 108, face 107 & phase & maintien & -.54 & $y=-0,000$ & $a+24,14$ & du temps \\
\hline P 108 , face 107 & phase de & hargement & -.64 & $y=-0,000$ & $\sigma+24,09$ & du temps \\
\hline
\end{tabular}

Tableau II. - Coefficients de régression linéaire des courbes de déformation et charge au niveau des piliers.

Tableau II. - Coefficients of linear regression of deformation curves and pillars loading.

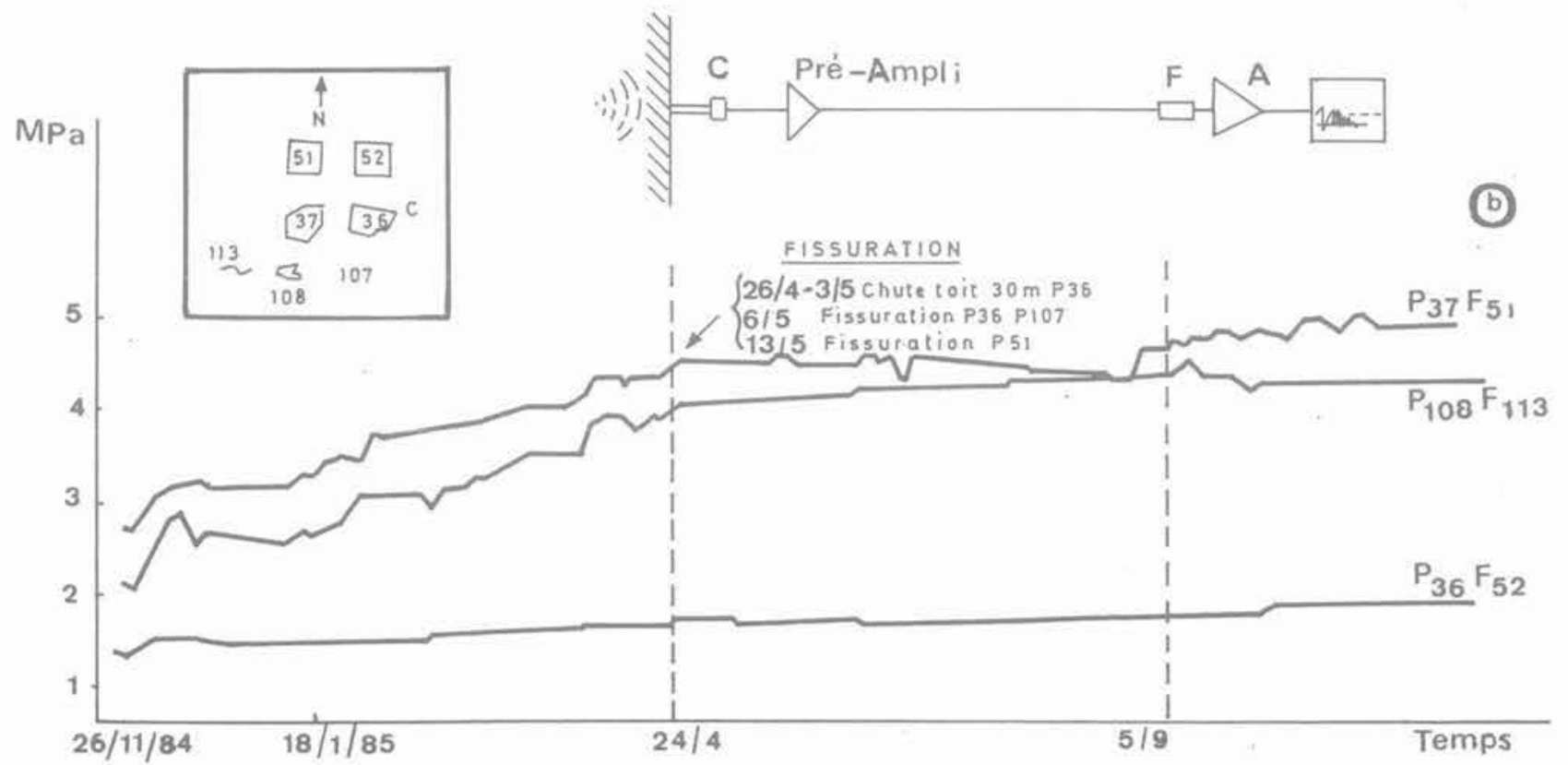

Fig. 2. - a) Tableau synoptique de la chaîne d'enregistrement acoustique.

$C$ : accéléromètre, préamplificateur - $F$ : filtre - $A$ : amplificateur, compteur.

b) Relation entre la surcharge et le temps.

A gauche, diagramme simplifié montrant la position des piliers.

Fig. 2. - al Block diagram of acoustic emission monitoring system.

$C$ : accelerometer, preamplifier - $F$ : filter - A : amplifier, counter.

b) Pillar compression versus time.

On the left, simplified diagram showing the position of pillars. 
acier dont l'extrémité était enfoncée dans un trou préalablement foré au quart de la tête du pilier 36 ; face est. Ce détecteur était relié à un préamplificateur de charge ;

- d'un filtre (passe-haut $600 \mathrm{~Hz}$ ) et d'un amplificateur.

Les signaux dépassant un seuil pré-réglé (100 mV) sont enregistrés à intervalles de temps réguliers (90 secondes) par un enregistreur à plume qui fonctionne en continu. L'ensemble de la chaîne était alimenté par batteries. Un câble de 100 mètres établissait une liaison entre le préamplificateur de charge et le compteur numérique dans un terminal.

\section{MISE EN SURCHARGE DU CHANTIER}

La mise en surcharge progressive du chantier a commencé le 26 novembre 1984 et s'est poursuivie jusqu'à la fin du mois d'avril (figure 2).

Une légère remise en surcharge a été de nouveau appliquée du 5 septembre 1985 au 28 octobre 1985

L'apparition des fissures sur les piliers est mentionnée sur la figure 2 ainsi que leur emplacement. Toutefois il convient de noter que les dates sont approximatives en raison de l'espacement des visites dans la carrière.

\section{EXPLOITATION ET INTERPRÉTATION DES RÉSULTATS}

\section{1. Évolution de l'émission acoustique en fonction du temps}

Nous présentons les relevés acoustiques effectués entre le 27 mars et le 29 juin 1985, soit une période de trois mois au cours de laquelle ont pu être observés les effets de la fin de la période de remblayage.

Les trois premiers mois n'ont pas été pris en considération en raison de parasites électriques qui interféraient sur la chaîne acoustique. Ceux-ci étaient dus au champ électromagnétique délivré par l'ensemble des autres appareils de mesure. La nécessité est donc apparue de relier l'appareillage à une alimentation sur batterie.

La zone de détection de l'accéléromètre implanté sur le pilier 36, face EST était de l'ordre d'une trentaine de mètres: l'endommagement interne puis lapparition des fissures sur les piliers 107 et 51 distants d'une dizaine de mètres et proches du pilier 36 ont été par conséquent pris en considération par le capteur.

Sur la figure 3 ont été reproduits en fonction du temps :

- le nombre de coups par heure $N_{c / \mathrm{h}}$ :

- l'évolution des contraintes du pilier 37, face 51 , très proche du pilier 36 , le vérin implanté sur le pilier 36, face 52 n'ayant pas accusé de variations notables de pression, peut-être en raison de l'hétérogénéité des faces de ce pilier.

On constate sur la figure $3 \mathrm{a}$ relative au mois de mars que le lendemain de la reprise de la surcharge interrompue du 19 au 27 mars, une libération brutale d'activité très importante le 29 mars (15 032 coups) s'est manifestée, suivie d'une absence presque totale de phénomènes aléatoires, sporadiques et de faible intensité (sauf les 12, 15, 20 et 23 avril).

On peut supposer dans ce cas qu'un tel type d'activité peut être lié à une fracture importante des roches du toit dans la zone du pilier 36 .

Quelques jours après l'arrêt de la surcharge en surface, le 24 avril, une importante émission est apparue à nouveau, le 29 avril (15 992 soit $10^{4}$ coups) dont la durée fut très courte (20 minutes) ; la fin de cette phase a pu coïncider avec une chute de toit à 30 mètres du pilier 36 suivie de l'apparition de fissures sur les piliers 36 et 107 d'après les observations visuelles du 26 avril, des 3 et 6 mai.

La figure $3 b$ représente l'évolution de l'émission acoustique au cours du mois de mai. Sur ce graphique, on voit apparaître un mécanisme de fissuration qui se différencie des décharges brutales précédentes dans ce sens qu'il se caractérise par une microactivité $\left(10^{2}\right.$ à $10^{3}$ coups par heure) qui s'étend sur une longue durée; cette phase se termine par un pic qui représente un nombre de coups élevé.

Ce phénomène peut durer deux à trois jours ainsi que l'atteste l'apparition en surface de fissures (pilier 51, le 13 mai). Dans ce cas, il s'agirait d'un mécanisme de propagation lente de la fissure. De telles manifestations ont été observées par TROMBIK lors de la surveillance sismo-acoustique de mines de charbon en Pologne.

Après une accalmie de près de trois semaines en juin (figure $3 \mathrm{c}$ ) une activité acoustique discrète révêle une progression de la fissuration dans les mêmes secteurs entre le 17 juin et le 26 juin.

\subsection{Corrélation entre les déformations et l'émission acoustique}

Afin de mieux approfondir les relations entre les paramètres physiques et mécaniques qui président à la phase d'endommagement, il est apparu souhaitable de représenter d'abord l'évolution des déformations des piliers considérés soit en fonction de la phase de chargement, soit en fonction du temps lorsqu'on ne disposait pas des mesures de contraintes, et ensuite l'évolution des déformations en fonction de l'émission acoustique.

\subsection{1. Évolution des déformations en fonction de la charge}

Des comparaisons ont pu être établies entre le comportement de certains piliers et la charge en surface, à l'aide de coefficients de corrélation.

En effet on voit sur le tableau II que le coefficient de corrélation de Bravais Pearson est d'autant plus élevé 


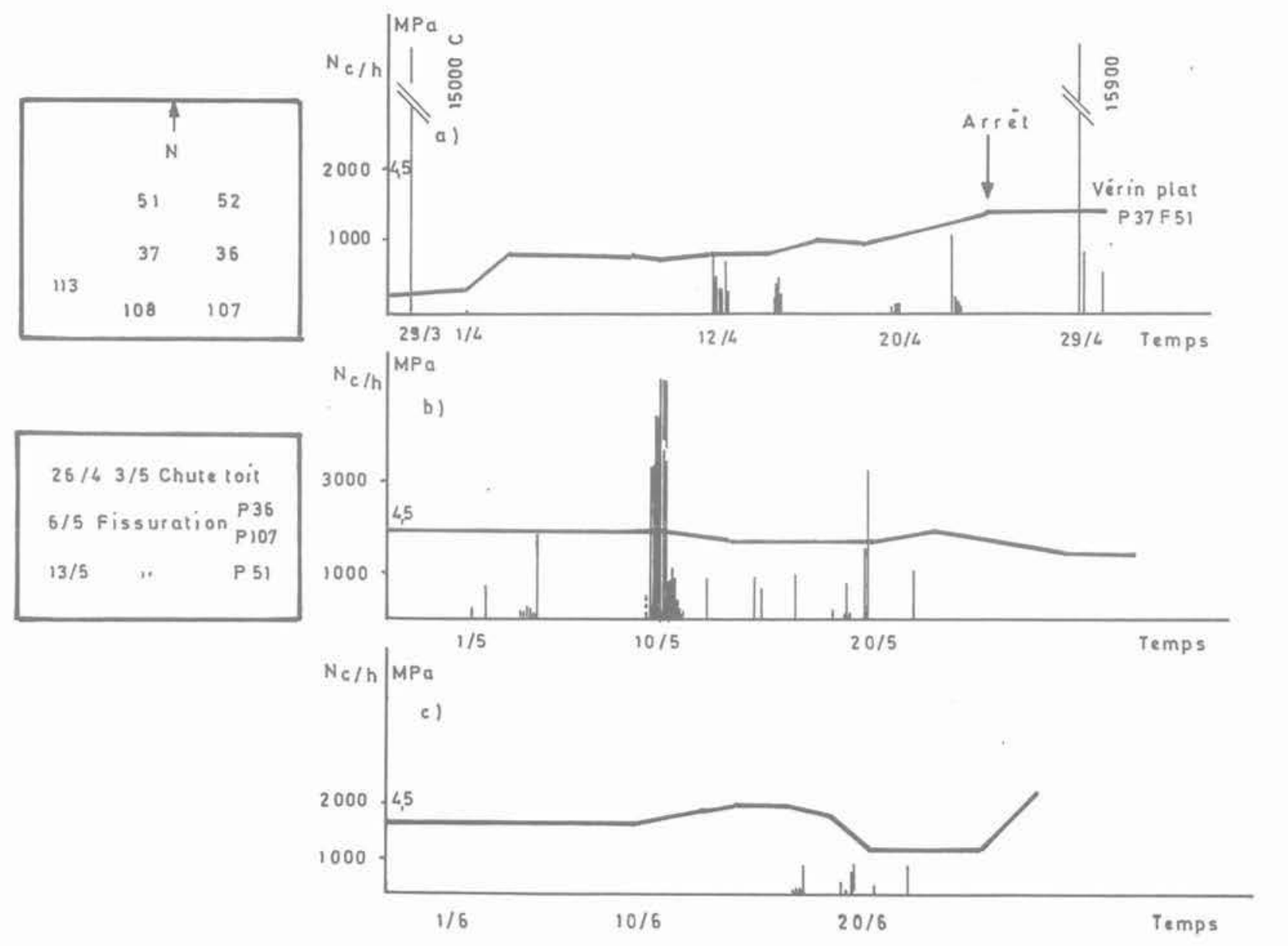

Fig. 3. - Relation entre le taux d'émission acoustique, le temps et la charge (p. 37, f. 51) :

a) au cours du mois d'avril 1985;

b) au cours du mois de mai 1985;

c) au cours du mois de juin 1985.

Fig. 3. - Relation between acoustic emission rate, time and pillar compression (p. 37, f. 51):

a) during april 1985;

b) during may 1985 ;

c) during june 1985 .

que la contrainte relevée sur le pilier à l'état initial est importante.

Le pilier qui a paru le mieux refléter les différentes phases de chargement en surface est le pilier 108 , face 107 (phase de chargement - phase de maintien phase de rechargement : Tableau II).

La pente de la droite de régression est la plus faible lorsque la phase de remblayage est arrêtée. Néanmoins si on se reporte à la valeur du coefficient de corrélation celui-ci n'est pas nul. Ce fait est vraisemblablement dû à une redistribution des contraintes dans la zone considérée.

\subsection{2. Évolution des déformations en fonction de l'émission acoustique}

L'analyse simultanée de l'évolution des paramètres mécaniques et physiques permet de localiser la zone fissurée. L'examen de l'ensemble de l'évolution des déformations au niveau des piliers 36,108 et 37 révèle une fissuration de certains d'entre eux lorsqu'il se produit une diminution progressive des déformations qui s'étend sur une durée d'une dizaine de jours. Les exemples qui suivent illustrent ce fait.

Le pilier 36, face 52 sur lequel est implanté l'accéléromètre accuse en son milieu une brusque chute des déformations le $1^{\text {er }}$ avril (figure 4). Lorsqu'on superpose l'histogramme des microbruits (nombre de coups cumulés sur une période de 24 heures) sur le graphique des déformations (figure 5) on observe :

- qu'une importante émission acoustique précède la diminution des déformations le 29 mars. Elle peut être considérée comme une prévision de l'affaissement d'une zone qui a atteint quelques hectares;

- qu'une zone d'activité sismo-acoustique se manifeste du 17 avril au 29 avril correspondant à une 


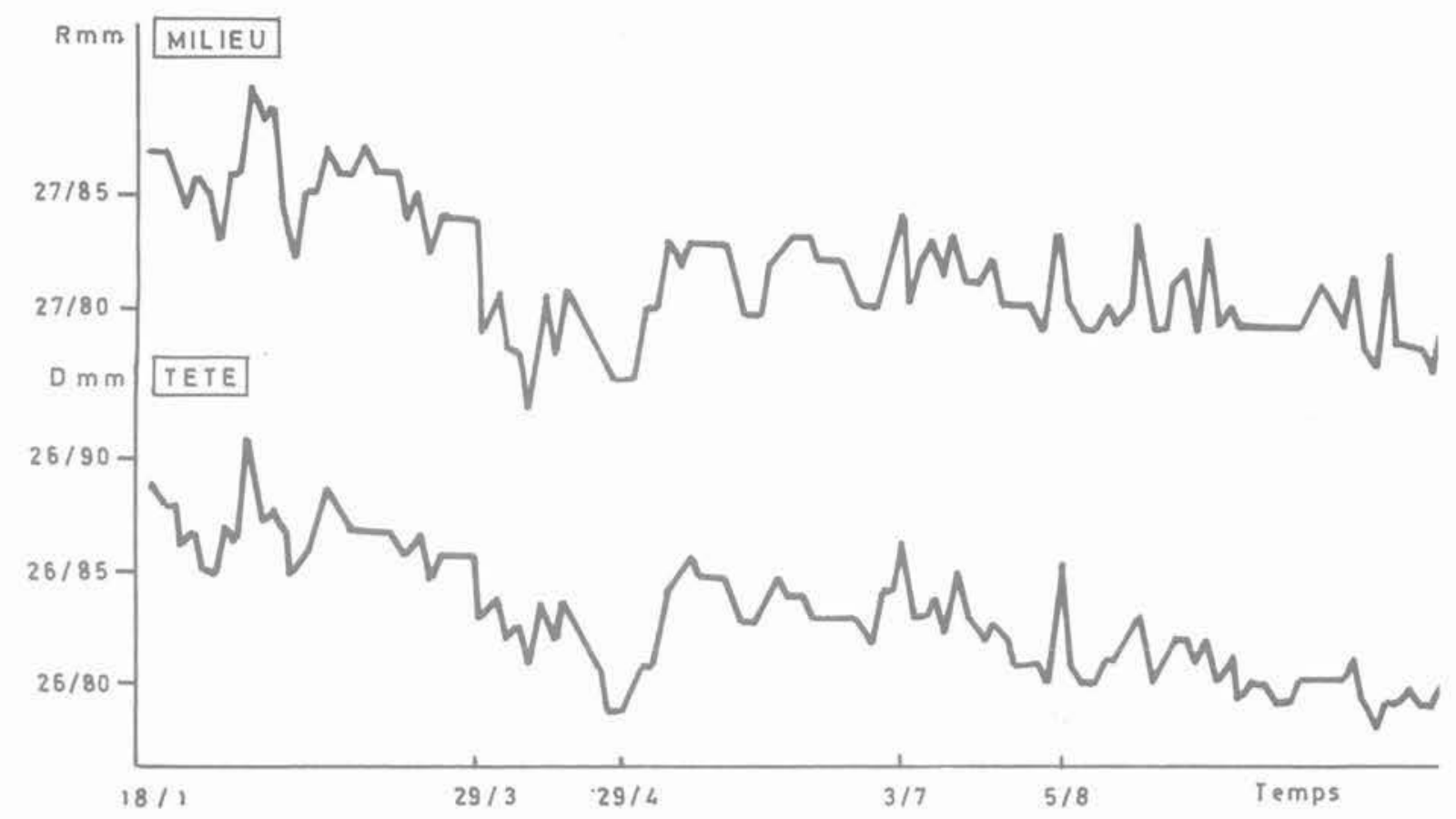

Fig. 4. - Évolution des racourcissements du pilier 36, face 52 du 18 janvier 1985 au 31 décembre 1985. Fig. 4. - Deformations (extensometer p. 36, f. 52) from january the 1st, 1985 to december the 31 th, 1985.

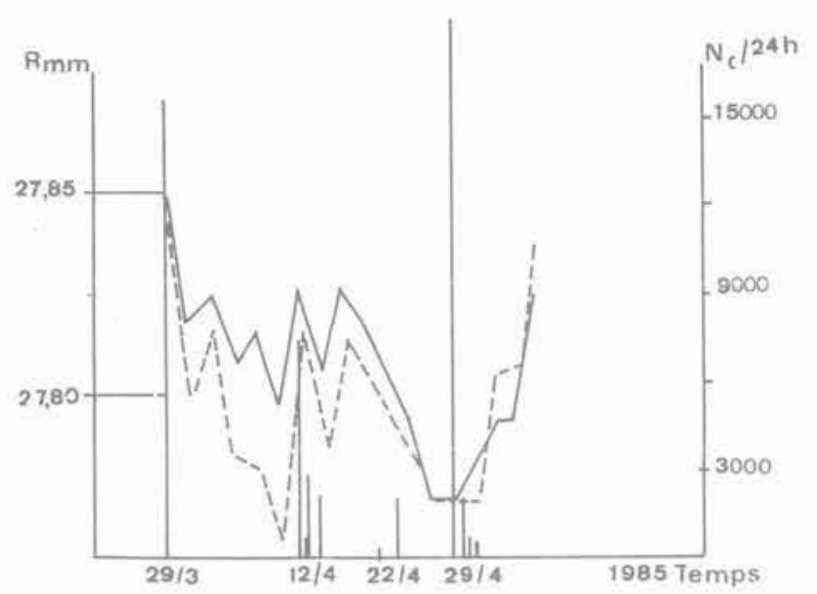

Fig. 5. - Relation entre le taux d'émission acoustique par 24 heures

et les racourcissements du p. $36, f .52$

du 29 mars 1985 au 29 avril 1985.

Fig. 5. - Relation between acoustic emission rate during 24 hours and deformations ( $p .36, f .52$ ) from march the 29th, 1985 to april the 29th, 1985.

décroissance spectaculaire des déformations. Un pic d'un nombre de coups très élevé $(18$ 200) laisse supposer la fissuration des piliers 36,52 et 107 ; ceci a été contrôlé visuellement le 6 mai.

De même lorsqu'on superpose l'histogramme acoustique sur le graphique des déformations du pilier 37 . face 51 , on observe une intense activité dont le maxi- mum (63000 coups) correspond à la valeur la plus faible des déformations le 11 mai, date vraisemblable de la fissuration du pilier 51 que le contrôle visuel a mentionné le 13 mai.

\section{CONCLUSIONS}

Dans le cadre de cette étude, l'interprétation des résultats sismo-acoustiques de la carrière peut s'avérer fiable en ce qui concerne l'évaluation du risque de fissuration des piliers et éventuellement de l'effondrement brutal d'une zone instable.

Au vu de l'ensemble des données, il se dégage quelques aspects positifs, à savoir :

- l'existence dans le domaine de la mécanique de la rupture de deux types de signaux : des signaux caractérisés par une activité composée de salves d'amplitudes très importantes, de courte durée, apparaissant d'une façon aléatoire, apériodique. Une telle activité est très probablement le résultat de fractures périodiques du toit. Un deuxième type de signal caractérisé par une microactivité s'étendant sur plusieurs jours et qui semble lié à une progression lente de la fissuration:

- l'existence d'une relation entre l'évolution des déformations d'un pilier et l'activité acoustique qui permet de localiser la source des microbruits.

L'analyse des microbruits permet par conséquent de mettre en évidence des zones instables et dangereuses. Elle constitue un moyen d'investigation de la stabilité de la carrière. 


\section{BIBLIOGRAPHIE}

HARDY H.R. Jr (1975 b), Evaluating the stability of geologic structures using Acoustic emission in monitoring structural integrity by acoustic emission. S.T.P. 571. American Society for testing and materials, Philadelphia, pp. 90-106.

ANTSYFEROV M.S.. PETROSIAN A.E., ARABADZHEV A.M., IVANOV U.S. and KLEMPER K.S. (1974), The seismic acoustic method of forecasting the danger of outburst. Symposium on coal and Gas outbursts, Donatz, 1974, United Nation.

JAROSZEWSKA A. (1982), Investigation of the waveguide properties of a borehole and their use for acous- tic measurements in situ, Archives of acoustics 7, 2, pp. 119-142.

REYMOND M.C., CLERGEOT A., LUMEAU B., PERROT F. (1984), Acoustic emission in coal and masonry tunnels. Proceeding third conference on acoustic emission/microseismic activity in geologic structures and materials, Pennsylvania State University, october 1981. Trans. Tech. Publication Clausthal Germany, vol. 8, pp. 117-130.

LASOCKI, "Statistical method of estimating the probability of occurence of strong mining shocks in the longwall region, Pubs. Inst. Geoph. Pol. Ac. Sci. M 5/155/1982. 